\section{New transgenic mouse model of spontaneous autoimmune arthritis}

Rankin and colleagues' paper in The Journal of Immunology describes a new transgenic mouse model of spontaneous autoimmune arthritis, in which the disease process is driven by recognition of a surrogate self antigen by autoreactive $\mathrm{CD}^{+}{ }^{+} \mathrm{T}$ cells. They hope that future studies using this model will provide insight into the mechanisms by which particular MHC class II alleles confer susceptibility to rheumatoid arthritis in humans, and allow evaluation of new potential treatments.

Briefly, the authors crossed two lineages of mice: TS1 mice express a T-cell-receptor transgene that recognizes a hemagglutinin epitope (the surrogate self antigen), and HACII mice express hemagglutinin on antigenpresenting cells (APCs) under the control of a MHC class II promoter. Most TS $1 \times \mathrm{HACll}$ mice spontaneously develop adult-onset arthritis, characterized by lung pathology and a localized immune response in lymph nodes that drain major joints; a few develop severe, multifocal, autoimmune disease. Importantly, TS1 1 HACll mice engineered to lack B cells also developed arthritis, which indicated that disease development was not mediated by autoantibodies or B-cell activation.

In TS1 1 HACll mice, arthritis seems to result from chronic overproduction of proinflammatory cytokines, including interleukin 17 , by autoreactive but hyporesponsive CD4 ${ }^{+}$ $T$ cells. These $T$ cells accumulate despite multiple pathways of tolerance induction, and induce systemic APC activation. Activated APCs migrate to popliteal lymph nodes and initiate arthritis via interactions with $\mathrm{CD}^{+}$ $T$ cells, but usually do not mediate pathology in other organs.

Original article Rankin AL et al. (2008) $\mathrm{CD} 4^{+} \mathrm{T}$ cells recognizing a single self-peptide expressed by APCs induce spontaneous autoimmune arthritis. J Immunol 180: 833-841

\section{Sphingosine 1 phosphate is a key mediator of inflammation in primary Sjögren's syndrome}

Sphingosine 1 phosphate (S1P) is an extracellular lysophospholipid signaling molecule that is involved in a diverse range of cellular processes, including inflammation and $\mathrm{T}$ cell development. Signaling between S1P and epithelial cells is mediated by a G-protein-coupled receptor, $\mathrm{S} \mathrm{P}_{1}$. As epithelial cells of labial salivary glands are thought to be actively involved in the inflammatory process observed in primary Sjögren's syndrome, Sekiguchi and colleagues studied the role of $\mathrm{S}_{1} \mathrm{P}_{1}$ signaling in the pathogenesis of this disease.

$\mathrm{S} \mathrm{P}_{1}$ was significantly overexpressed in infiltrating mononuclear cells in salivary gland specimens from patients with Sjögren's syndrome. $\mathrm{CD}^{+} \mathrm{T}$ cells in the peripheral blood of patients and healthy control individuals also expressed $\mathrm{S}_{1} \mathrm{P}_{1}$. The application of S1P plus monoclonal anti-CD3 antibody enhanced $\mathrm{CD}^{+}$T-cell proliferation; S1P alone increased IFN- $\gamma$ secretion by $\mathrm{CD}^{+}{ }^{+} \mathrm{T}$ cells, but to a greater extent in patients than in controls. In vitro experiments showed that both IFN- $\gamma$ and S1P induce Fas (CD95)-mediated apoptosis in salivary gland epithelial cells and, at high levels of S1P and in advanced stages of Sjögren's syndrome, increase the secretion of IL-6-a cytokine known to be overexpressed in many autoimmune diseases-by ductal epithelial cells.

The authors conclude that $\mathrm{S}_{1} \mathrm{P}_{1}$ signaling seems to have an important role in the pathogenesis of primary Sjögren's syndrome, and contributes to several mechanisms of inflammation in both epithelial and mononuclear cells. Therapies that regulate $\mathrm{S}_{1} \mathrm{P}_{1}$ signaling might be effective in patients with this disease.

Original article Sekiguchi M et al. (2008) Role of sphingosine 1-phosphate in the pathogenesis of Sjögren's syndrome. J Immunol 180: 1921-1928

\section{Successful treatment of collagen-induced arthritis with cell-cycle inhibitors}

Proliferation of synovial fibroblasts causes pannus formation in joints affected by rheumatoid arthritis (RA). Antiproliferative therapies, such as cyclin-dependent kinase (CDK) inhibitors, halt cell division and could potentially suppress pannus formation. Sekine and colleagues, therefore, investigated the effects of two smallmolecule CDK inhibitors - the broad-spectrum CDK inhibitor alvocidib, and a novel, selective, CDK4 + CDK6 inhibitor - on collagen-induced arthritis (CIA) in mice. 